\title{
Myocardial expression of heat shock protein 70i protects early postoperative right ventricular function in cyanotic tetralogy of Fallot
}

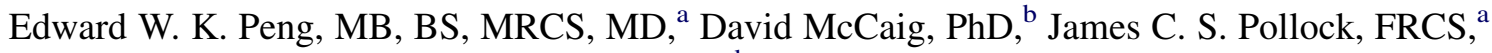 \\ Kenneth MacArthur, FRCS, ${ }^{\mathrm{a}}$ Fiona Lyall, PhD, ${ }^{\mathrm{b}}$ and Mark H. D. Danton, MD, FRCS(CTh) ${ }^{\mathrm{a}}$
}

\begin{abstract}
Background: Right ventricular dysfunction occurs after tetralogy of Fallot repair and may relate to greater myocardial vulnerability to ischemia-reperfusion injury in cyanotic patients. The inducible form of heat shock protein 70 (HSP-70i), a molecular chaperone, is upregulated in response to cellular stress and limits myocardial injury against ischemia-reperfusion. We evaluated the myocardial expression of HSP-70i and its relation to right ventricular function and clinical outcome in patients with tetralogy of Fallot undergoing corrective surgery.
\end{abstract}

\begin{abstract}
Methods: Twenty patients with tetralogy of Fallot were studied: 10 cyanotic (group Cy) and 10 noncyanotic (group noCy). Western blot was used to quantify HSP-70i from resected right ventricular outflow tract myocardium at baseline and subsequent ischemic time. Biventricular function was quantified by tissue Doppler echocardiography and compared with that of 15 age-matched healthy children. Postoperative systemic perfusion was assessed by mixed venous oxygen saturation, oxygen extraction ratio, and lactate.
\end{abstract}

Results: Group Cy had thicker septum (median 0.85 vs $0.66 \mathrm{~cm} ; P=.01$ ) and longer crossclamp time (median 100.0 vs 67.5 minutes; $P=.004$ ). There were no difference in HSP-70i between groups at baseline (4.12 vs 3.44 relative optical density; $P=.45$ ) or subsequent ischemic time. Preoperative biventricular systolic function was reduced in patients with tetralogy compared with controls with further postoperative right ventricular impairment. Group Cy had higher troponin-I levels (median $16.5 \mathrm{vs} 11.1 \mathrm{ng} / \mathrm{mL} ; P=.04$ ) and inotrope scores (14.0 vs $6.5 ; P=.05)$ but no differences in ventricular function, mixed venous oxygen saturation, oxygen extraction ratio, and lactate between groups. In group $\mathrm{Cy}$, baseline HSP-70i correlated with better postoperative right ventricular function (rho $=0.80 ; P=.009$ ), mixed venous oxygen saturation (rho $=0.68 ; P=.04$ ), and oxygen extraction ratio (rho $=-0.71 ; P=.03$ ). These relationships were absent in group noCy.

Conclusions: The association of HSP-70i expression with improved right ventricular function and systemic perfusion suggests an important cardioprotective effect of HSP-70i in cyanotic tetralogy of Fallot. (J Thorac Cardiovasc Surg 2011;141:1184-91)

Patients with congenital heart disease in whom the right ventricle (RV) is exposed to cyanosis and pressure overloading, as in tetralogy of Fallot (TOF), are prone to RV dysfunction after corrective surgery. The cause is multifactorial and includes residual hemodynamic load from pulmonary stenosis or regurgitation, conduction abnormalities, and direct surgical trauma. In addition, surgical repair exposes the myocardium to ischemic stress during cardiopulmonary bypass. Controversy exists as to whether the chronically hypoxic myocardium is more or less tolerant

From the Department of Cardiac Surgery, ${ }^{a}$ Royal Hospital for Sick Children, and the Department of Maternal \& Fetal Medicine Section, ${ }^{\mathrm{b}}$ Institute of Medical Genetics, Yorkhill, Glasgow, United Kingdom.

Funded by Yorkhill Children Foundation, Glasgow, United Kingdom.

Disclosures: Authors have nothing to disclose with regard to commercial support.

Read at the 90th Annual Meeting of The American Association for Thoracic Surgery, Toronto, Ontario, Canada, May 1-5, 2010.

Received for publication May 3, 2010; revisions received Jan 1, 2011; accepted for publication Jan 24, 2011; available ahead of print March 14, 2011.

Address for reprints: Mark H. D. Danton, MD, FRCS(CTh), Department of Cardiac Surgery, Royal Hospital for Sick Children, Dalnair Street, Glasgow G3 8SJ, United Kingdom (E-mail: danton.mark@googlemail.com).

0022-5223/\$36.00

Copyright $($ C 2011 by The American Association for Thoracic Surgery doi:10.1016/j.jtcvs.2011.01.047 of ischemia-reperfusion injury than is the normoxic myocardium. However, the adequacy of myocardial protection during corrective TOF repair has been an area of concern with evidence of oxygen-mediated free radical injury during surgery and inhomogeneous distribution of cardioplegic solution in a hypertrophied RV mass. ${ }^{1-4}$

Heat shock proteins (HSPs) are endogenous proteins that maintain cell homeostasis against various forms of stress as a cellular adaptive response. These proteins are induced by a wide variety of stressors including hyperthermia, ischemia, and oxidative and cytokine-mediated stress to provide a broad cytoprotective function. ${ }^{5,6}$ The 70-kDa HSP (HSP70 ) is the most widely studied of the family in the context of cardiovascular disease. ${ }^{5,6}$ The inducible form of HSP-70 (HSP-70i, also known as HSP-72) has been shown to limit myocardial ischemia-reperfusion injury and modulate the inflammatory response to cardiopulmonary bypass in both clinical and experimental studies. ${ }^{5-7}$ In children undergoing surgery for congenital heart disease, upregulation of HSP72 during cardiopulmonary bypass was associated with reduced markers of myocardial injury. ${ }^{8}$

Hypoxic stress in animal models induces various cellular adaptive pathways as a mechanism to minimize cellular 

Abbreviations and Acronyms
HSP $=$ heat shock protein
HSP-70 $=70-\mathrm{kDa}$ heat shock protein
$\mathrm{LV} \quad=$ left ventricle (ventricular)
POD = postoperative day
$\mathrm{RV}=$ right ventricle (ventricular)
RVOT = right ventricular outflow tract
$\mathrm{S}_{\mathrm{a}} \mathrm{O}_{2}=$ systemic arterial oxygen saturation
$\mathrm{S}_{\mathrm{v}} \mathrm{O}_{2}=$ mixed venous oxygen saturation
TOF $=$ tetralogy of Fallot

procedure. Direct RV and left ventricular (LV) pressures were measured intraoperatively after repair.

\section{Protein Analysis}

All myocardium resected from the RVOT as part of the repair was collected for protein analysis. Muscle was immediately snap-frozen in liquid nitrogen after resection and segregated according to the ischemic time points and storage at $-70^{\circ} \mathrm{C}$. The onset of aortic crossclamping denoted the beginning of ischemic time.

Extraction of cell lysate from tissues. Tissue homogenization was performed with a Polytron PT 1600 E homogenizer (Kinematica AG, Lucerne, Switzerland). ${ }^{13}$ Homogenates were centrifuged at $5000 \mathrm{~g}$ at $4{ }^{\circ} \mathrm{C}$ for 10 minutes. The supernatant, containing the total cell protein (both particulate and cytosolic fractions) was extracted. Bradford protein assay was used to quantify the total protein concentration. ${ }^{14}$

Western blot analysis. Western blot was performed according to protocol as previously described. ${ }^{13}$ The nitrocellulose membrane was incubated first in primary antibody solution (rabbit HSP-72 polyclonal antibody; Assay Designs, Inc, Ann Arbor, Mich; catalog No. SPA-812) and subsequently in secondary antibody solution (donkey antirabbit; Abcam plc, Cambridge, United Kingdom; catalog No. ab7084). Manufacturer (Assay Designs, Inc) testing did not show any cross-reaction between primary HSP-72 antibody with recombinant form of constitutive HSP-70. Homogenized placental villous tissue, known to contain HSP-70i, was used as a reference sample and internal control. The density of the protein bands was quantified by a computerized densitometer (BioRad GS-700, Quantity One software; Bio-Rad Laboratories, Hercules, Calif). HSP level was measured as relative optical density to internal control.

The baseline period was defined as 0 to 15 minutes after aortic crossclamping and before repeat administration of cardioplegic solution. Baseline HSP-70i protein density was calculated from mean HSP-70i in the RV myocardium harvested during this period and considered to represent the preoperative HSP-70i in the myocardium. Subsequent muscle resected after this period was grouped into 15-minute intervals during aortic crossclamping to examine any effect of ischemic time on HSP expression.

\section{Postoperative Monitoring}

Postoperatively, serum lactate level, mixed venous oxygen saturation $\left(\mathrm{S}_{\mathrm{v}} \mathrm{O}_{2}, \%\right)$ and troponin-I were measured concurrently with echocardiography. Inasmuch as $\mathrm{S}_{\mathrm{v}} \mathrm{O}_{2}$ is a function of systemic arterial oxygen saturation $\left(\mathrm{S}_{\mathrm{a}} \mathrm{O}_{2}\right)$, which can be affected by various factors including right-to-left intracardiac shunt and lung disease, oxygen extraction ratio $\left(\mathrm{OER}=\left[\mathrm{S}_{\mathrm{a}} \mathrm{O}_{2}-\right.\right.$ $\left.\mathrm{S}_{\mathrm{v}} \mathrm{O}_{2}\right] / \mathrm{S}_{\mathrm{a}} \mathrm{O}_{2}$ ) represents a better indicator of systemic perfusion. ${ }^{15}$ Oxygen saturation was measured from concurrent blood sampling taken from the peripheral arterial line and central venous catheter in the superior vena cava. ${ }^{15}$ Inotrope score was calculated on the basis of Wernovsky and associates. ${ }^{16}$ Duration of ventilation and intensive care unit stay was recorded.

\section{Statistical Analysis}

Statistical analysis was performed using Minitab Statistical Software Release 14.2 (Minitab Inc, State College, Pa). Nonparametric data (expressed in median \pm interquartile range, Q1-Q3) was analyzed using the Mann-Whitney $U$ test, Wilcoxon matched pair, or Kruskal-Wallis test for continuous variables and Fisher's exact test for categorical variables. The strength of the relationship between 2 variables was tested using the Spearman rank correlation (rho).

\section{RESULTS}

There were no demographic difference between groups $\mathrm{Cy}$ and noCy in terms of age, gender, and body weight. Group Cy had a higher hematocrit level, thicker septal
Standard repair was performed by closure of the ventricular septal defect and resection of RV infundibular muscles via transtricuspid and transperate hypothermia and blood cardiopleg. and fractional inspired oxygen was adjusted to maintain adequate oxygenation while avoiding hyperoxia (median arterial oxygen tension $8.0 \mathrm{kPa}$ in group Cy vs $12.1 \mathrm{kPa}$ in group noCy; $P=.2$ ). Epicardial echocardiography was routinely performed to exclude any residual lesion at the end of the 
TABLE 1. Perioperative characteristics between group Cy and group noCy

\begin{tabular}{|c|c|c|c|c|}
\hline & $\begin{array}{c}\text { Group Cy } \\
(\mathbf{n}=\mathbf{1 0})\end{array}$ & $\begin{array}{c}\text { Group noCy } \\
\quad(\mathbf{n}=\mathbf{1 0})\end{array}$ & $P$ value & $\begin{array}{c}95 \% \text { CI of } \\
\text { median difference }\end{array}$ \\
\hline \multicolumn{5}{|l|}{ Preoperative factors } \\
\hline Age (mo) & $22.1(11.8-24.3)$ & $16.4(6.8-23.4)$ & .36 & $(-3.8,16.3)$ \\
\hline Gender (n, \%) & 6 males $(60 \%)$ & 5 males $(50 \%)$ & 1.00 & - \\
\hline Weight (kg) & $9.2(8.6-12.2)$ & $10.5(7.9-11.0)$ & .94 & $(-2.2,3.2)$ \\
\hline Hematocrit level (\%) & $47.0(40.8-50.0)$ & $38.1(37.0-40.9)$ & .01 & $(1.9,11.3)$ \\
\hline Oxygen saturation (\%) & $76(68-86)$ & $93(91-97)$ & .0002 & $(-26,-10)$ \\
\hline Blalock-Taussig shunt (n, \%) & $6,60 \%$ & $2,20 \%$ & .17 & - \\
\hline RV wall thickness (cm) & $0.70(0.58-0.83)$ & $0.65(0.57-0.82)$ & .91 & $(-0.13,0.16)$ \\
\hline IVS wall thickness (cm) & $0.85(0.70-0.94)$ & $0.66(0.54-0.75)$ & .01 & $(0.08,0.34)$ \\
\hline Sm-tricuspid annulus $(\mathrm{cm} / \mathrm{s})$ & $10.01(7.28-11.55)$ & $7.85(7.49-10.13)$ & .31 & $(-1.12,3.64)$ \\
\hline Sm-basal septum (cm/s) & $4.93(4.18-6.03)$ & $4.69(3.73-6.10)$ & .53 & $(-1.52,0.82)$ \\
\hline Sm-mitral annulus $(\mathrm{cm} / \mathrm{s})$ & $4.90(3.33-6.23)$ & $4.83(3.95-5.63)$ & .96 & $(-1.47,1.72)$ \\
\hline \multicolumn{5}{|l|}{ Intraoperative factors } \\
\hline Crossclamp time (min) & $100.0(75.0-130.0)$ & $67.5(61.8-76.0)$ & .004 & $(-58.0,-8.0)$ \\
\hline Bypass time (min) & $218.5(180.8-310.0)$ & $114.5(98.8-130.3)$ & .003 & $(63.0,182.0)$ \\
\hline Transannular patch use $(\mathrm{n}, \%)$ & $6,60 \%$ & $4,40 \%$ & .67 & - \\
\hline Subvalvular patch $(\mathrm{n}, \%)$ & $5,50 \%$ & $2,20 \%$ & .35 & - \\
\hline $\mathrm{RV} /$ systemic pressure ratio & $0.79(0.69-1.11)$ & $0.72(0.62-0.88)$ & .41 & $(-0.12,0.35)$ \\
\hline Baseline HSP-70i (R.O.D.) & $4.12(2.50-7.44)$ & $3.44(1.75-6.67)$ & .45 & $(-2.10,4.02)$ \\
\hline HSP-70i from all tissues (R.O.D.) & $3.82(2.59-7.53)$ & $3.17(1.40-6.65)$ & .39 & $(-1.97,2.74)$ \\
\hline \multicolumn{5}{|l|}{ Postoperative factors (POD 1) } \\
\hline Troponin-I (ng/mL) & $16.5(11.7-51.0)$ & $11.1(7.6-13.5)$ & .04 & $(0.1,37.6)$ \\
\hline Inotrope score & $14.0(9.5-16.3)$ & $6.5(0.8-14.9)$ & .05 & $(0.0,13.0)$ \\
\hline Hemoglobin (g/dL) & $12.2(11.4-14.0)$ & $11.5(10.4-13.6)$ & .41 & $(-1.2,2.1)$ \\
\hline $\mathrm{S}_{\mathrm{v}} \mathrm{O}_{2}(\%)$ & $65.4(55.5-71.3)$ & $66.5(61.8-68.3)$ & .91 & $(-9.0,7.0)$ \\
\hline Oxygen extraction ratio & $0.33(0.28-0.42)$ & $0.32(0.27-0.35)$ & .68 & $(-0.1,0.1)$ \\
\hline Oxygen excess factor $(\Omega)$ & $3.1(2.4-3.6)$ & $3.1(2.9-3.7)$ & .68 & $(-0.9,0.6)$ \\
\hline Lactate & $1.6(0.9-1.9)$ & $1.2(0.88-1.35)$ & .26 & $(-0.2,0.9)$ \\
\hline Sm-tricuspid annulus $(\mathrm{cm} / \mathrm{s})$ & $3.60(2.50-4.60)$ & $4.08(2.87-4.77)$ & .85 & $(-1.56,0.93)$ \\
\hline Sm-basal septum $(\mathrm{cm} / \mathrm{s})$ & $3.21(2.39-4.48)$ & $2.71(2.13-4.66)$ & .52 & $(-0.83,1.53)$ \\
\hline Sm-mitral annulus $(\mathrm{cm} / \mathrm{s})$ & $5.21(3.94-6.46)$ & $3.98(3.61-5.15)$ & .10 & $(-0.24,2.46)$ \\
\hline Restrictive RV physiology & $2,20 \%$ & $3,30 \%$ & 1.00 & - \\
\hline Ventilation time (h) & $141.0(58.7-195.2)$ & $82.0(19.1-121.3)$ & .14 & $(-7.7,122.7)$ \\
\hline Critical care stay (d) & $7.8(5.0-11.2)$ & $5.0(3.5-8.3)$ & .27 & $(-2.3,6.1)$ \\
\hline
\end{tabular}

All data are expressed in median (interquartile range). $C I$, Confidence intervals; $R V$, right ventricular; $I V S$, interventricular septum; $S m$, systolic myocardial velocity; $H S P$-70i, inducible form of heat shock protein 70; R.O.D., relative optical density; $P O D$ l, postoperative day $1 ; \mathrm{S}_{\mathrm{v}} \mathrm{O}_{2}$, mixed venous oxygen saturation.

wall, and longer bypass and crossclamp times. Group Cy required higher inotrope requirement on POD 1 and tended to require longer ventilation time and intensive care unit stay (Table 1).

\section{Preoperative and Postoperative Ventricular Function}

In comparison with healthy control subjects, patients with TOF had reduced systolic annular velocity preoperatively at the tricuspid annulus $(8.13 \mathrm{vs} 12.67 \mathrm{~cm} / \mathrm{s}$ in control; $P<.001)$, septum $(4.80$ vs $7.578 ; P<.001)$, and mitral annulus ( 4.83 vs $7.52 ; P<.001)$. There were no differences in preoperative systolic annular velocities between group Cy and group noCy (Table 1).

Postoperatively (day 1), systolic ventricular velocities were further reduced in the tricuspid annulus and basal septum in both group $\mathrm{Cy}$ and group noCy compared with preoperative velocities. $\mathrm{LV}$ velocities did not change significantly (Figure 1, $A$ and $B$ ). There was no association between postoperative RV velocities (tricuspid and septal) and preoperative oxygen saturation, hematocrit, aortic crossclamp time, troponin release, transannular patch use, ventriculotomy, degree of pulmonary regurgitation, or presence of restrictive $\mathrm{RV}$ physiology. There were no difference between postoperative systolic annular velocities or the presence of restritive RV physiology between group Cy and group noCy (Table 1).

At 1 week after surgery, in group $\mathrm{Cy}$, two thirds of patients had improved RV function (3.41 on POD 1 vs 4.88 $\mathrm{cm} / \mathrm{s}$ at 1 week; $P=.13)$ and $78 \%$ (7/9 patients) had improved septal function ( 3.45 on POD 1 vs $4.38 \mathrm{~cm} / \mathrm{s}$ at 1 week; $P=.20)$. In group noCy, two thirds of patients also had improved RV function ( 3.97 on POD 1 vs $4.98 \mathrm{~cm} / \mathrm{s}$ 


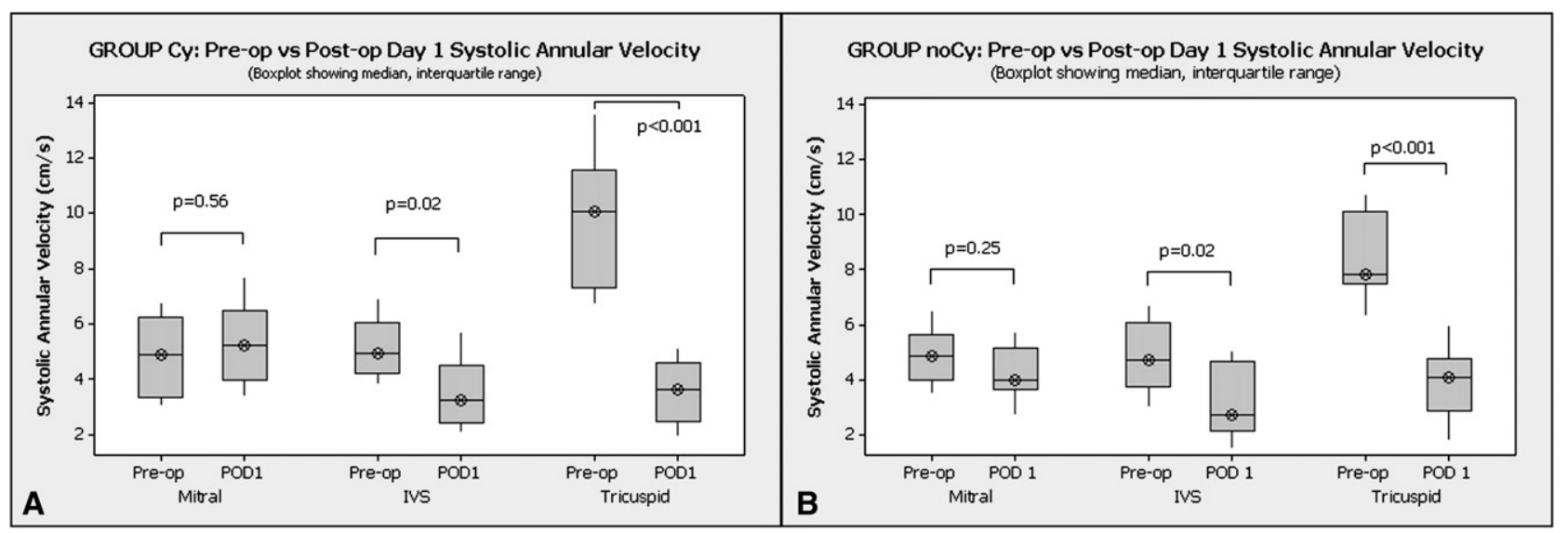

FIGURE 1. Systolic myocardial velocities were depressed on postoperative day 1 at the tricuspid annulus and interventricular septum but preserved at the mitral annulus in both group Cy (A) and group noCy (B). POD 1, Postoperative day; IVS, interventricular septum.

at 1 week; $P=.13$ ) and all had improved septal function ( 3.04 on POD 1 vs $5.77 \mathrm{~cm} / \mathrm{s}$ at 1 week; $P=.008$ ).

\section{Postoperative Troponin-I Release}

Group Cy had a higher troponin-I release on POD 1 (Table 1). Higher troponin-I release was associated with higher preoperative hematocrit level (rho $=0.63 ; P=.003$ ), longer aortic crossclamp time (rho $=0.50 ; P=.02$ ), and longer cardiopulmonary bypass time (rho $=0.50 ; P=.02$ ). There was no correlation between troponin-I and HSP-70i or the weight of the total muscles harvested in both groups.

\section{HSP-70i Expression in RVOT Muscles}

More RV muscles were resected in group Cy, although this difference did not reach statistical significance (738.7 vs $473.6 \mathrm{mg} ; P=.08$ ). HSP-70i was expressed in all muscles samples collected at different time points during the aortic crossclamping period (Figure 2). Mean HSP-70i from total RV muscles resected for each patient did not differ between groups $\mathrm{Cy}$ and noCy (3.82 vs 3.12 relative optical density; $P=.36$ ) (Figure 3,A). There was no difference in baseline HSP-70i or mean HSP-70i at different ischemic time points during aortic crossclamping between groups $\mathrm{Cy}$ and noCy. In each group, the median HSP-70i at later ischemic time points during aortic crossclamping was no different from the baseline period (Figure 3, B).
No significant relationship was found between baseline HSP-70i expression and preoperative variables: age, weight, preoperative oxygen saturation, hematocrit, and RV wall thickness.

\section{Relationship Between HSP-70i and Postoperative Ventricular Function and Outcome}

Overall in the whole cohort, an increased baseline HSP$70 \mathrm{i}$ was associated with better preserved RV function on POD 1 as expressed by higher annular velocities at the tricuspid (rho $=0.52 ; P=.02)$ and basal septum (rho $=0.55$; $P=.02$ ) (Figure 4). However, subgroup analysis revealed stronger relationships in group Cy: tricuspid (rho $=.80$; $P=.009)$ and basal septum (rho $=0.73 ; P=.025)$. In contrast, no correlation was seen between baseline HSP-70i and postoperative RV septal velocities in group noCy.

Higher baseline HSP-70i was associated with better $\mathrm{S}_{\mathrm{v}} \mathrm{O}_{2}$ (rho $\left.=.68 ; P=.04\right)$ and oxygen extraction ratio (rho $=-0.71 ; P=.03$ ) postoperatively in group $\mathrm{Cy}$, but not in group noCy (Figure 5). HSP-70i did not correlate with intraoperative postrepair RV/LV pressure, postoperative lactate level, inotrope score, and duration of ventilation or intensive care unit stay in either group.

\section{DISCUSSION}

Three main issues were addressed in this study: (1) baseline HSP-70i in an acyanotic versus cyanotic model

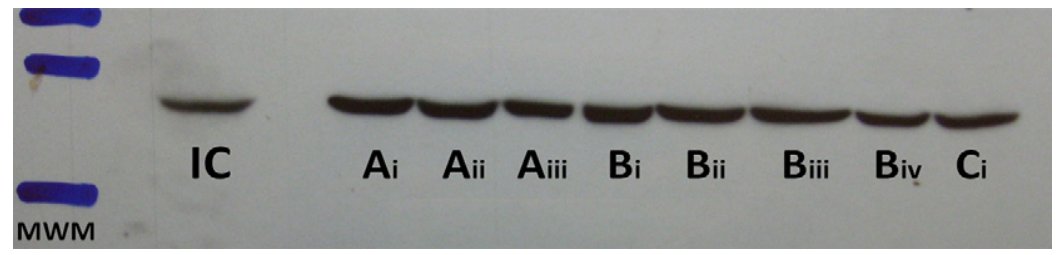

FIGURE 2. Western blot film: The density of each band represented the quantity of the inducible heat shock protein $70(H S P-70 i)$ for patients A, B, and C at separate ischemic time points (incremental from $i$ to $i i i$ ). $I C$, Internal control; $M W M$, molecular weight marker. 


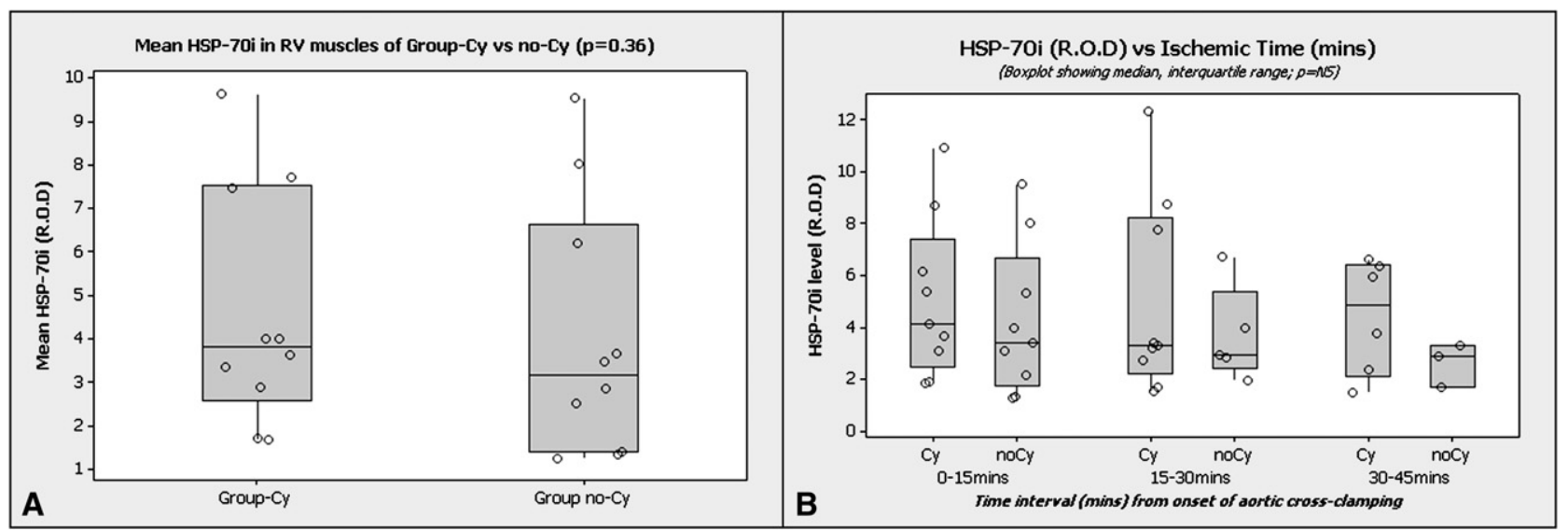

FIGURE 3. A, Boxplot (median, interquartile range) showing mean inducible heat shock protein 70 (HSP-70i) from total right ventricular $(R V)$ muscles resected for each patient in groups $\mathrm{Cy}$ and noCy $(P=.36)$. B, HSP-70i level at different ischemic time intervals during aortic crossclamping for groups $\mathrm{Cy}$ and noCy. R.O.D., Relative optical density.

(ie, HSP-70i in the chronic hypoxemic model), (2) HSP-70i changes during surgical repair (ie, HSP-70i in an acute on chronic hypoxemic model), and (3) the myocardial expression of HSP-70i and myocardial function. Earlier studies in postoperative repair of TOF have proposed that RV dysfunction was primarily related to diastolic relaxation whereas systolic contraction was relatively well preserved. ${ }^{12}$ In this study, we have shown that significant systolic RV dysfunction occurred early after surgery. This effect was equally apparent in both cyanotic and acyanotic patients, and a functional deficit remained 1 week postoperatively in one third of the patients. The degree of systolic RV dysfunction was not related to the presence of restrictive RV physiology or severity of pulmonary regurgitation, but correlated significantly with HSP-70i expression in the RVOT myocardium of cyanotic subjects. This relationship implies that early postoperative systolic RV dysfunction is associated with ischemia-reperfusion injury and its endogeneous adaptive pathway during surgery, rather the the degree of RV vol- ume or pressure overloading. Importantly, in cyanotic patients, an increased HSP-70i was also associated with better systemic tissue perfusion, suggesting an important cardioprotective role against the ischemia-reperfusion insult sustained during cardiopulmonary bypass.

\section{Cyanosis and the Myocardium}

In this study, we found that cyanotic patients in comparison with acyanotic had higher troponin levels postoperatively, increased inotrope requirement, but similar reduction in RV function after surgery. Troponin may increase because of greater muscle resection but, importantly, the cyanotic patients had longer crossclamp and cardiopulmonary bypass times than did the acyanotic group. There may also be an issue with impaired myocardial protection and greater susceptibility to ischemia-reperfusion injury in the cyanotic patients.

Controversy exists as to whether preoperative hypoxia renders the myocardium more or less susceptible to

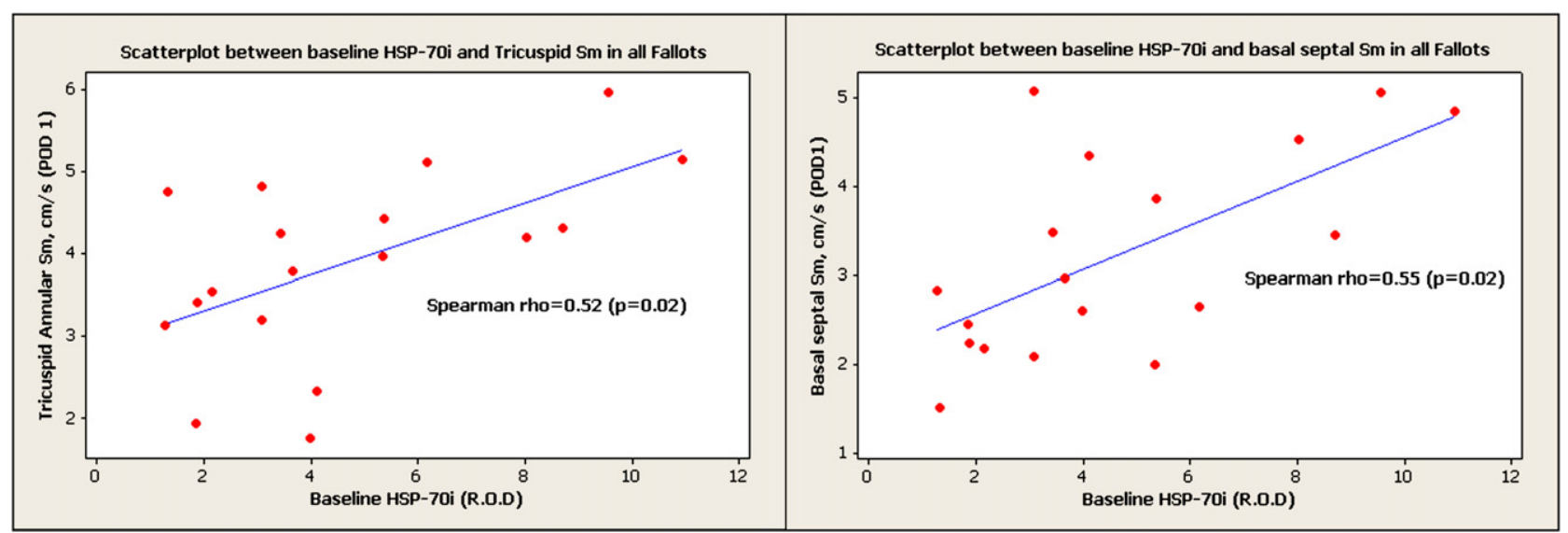

FIGURE 4. Scatterplot showing relationship between baseline inducible heat shock protein 70 (HSP-70i) expression and tricuspid annular and basal septal myocardial velocities $(\mathrm{Sm})$ on postoperative day $1(P O D$ 1) in all patients with tetralogy of Fallot. 


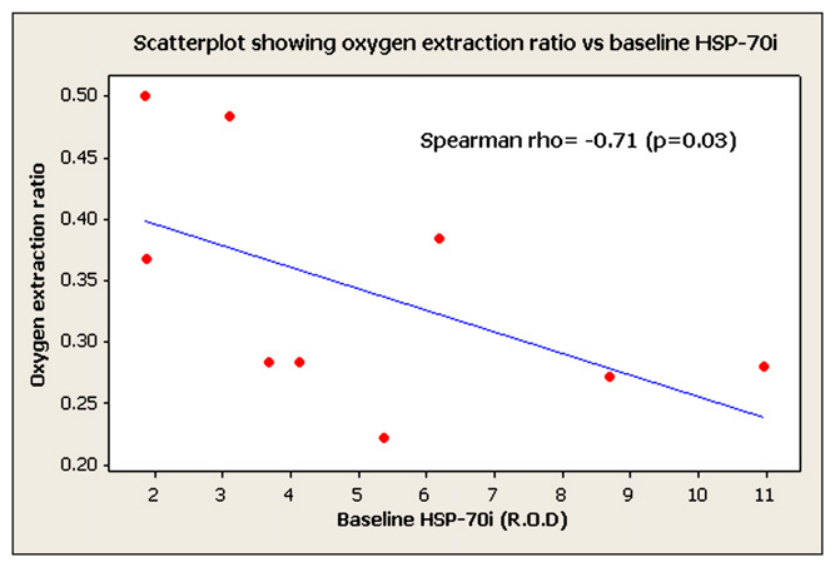

FIGURE 5. In Group-Cy, baseline inducible heat shock protein 70 (HSP$70 i)$ correlated inversely with oxygen extraction ratio suggesting better systemic perfusion with higher myocardial HSP-70 expression. R.O.D., Relative optical density.

ischemia-reperfusion injury sustained during bypass. Animal models suggest that chronic hypoxia confers tolerance to ischemia-reperfusion injury with improved functional LV recovery after ischemia-reperfusion stress. ${ }^{17}$ However, these animal models tend not to replicate exactly the pathophysiologic condition in TOF; specifically, the chronic hypoxic was induced by exposure to low oxygen tension and there was no RVOT obstruction. In another study, animal models in which the left atrium was anastomosed to a banded pulmonary artery (cyanotic-RVOT obstruction) were compared with patients with a banded pulmonary artery (RVOT obstruction alone) and those with a normal heart. ${ }^{18}$ An accelerated depletion of high-energy phosphates after cardioplegic arrest and reperfusion was seen in cyanotic-RVOT obstruction models, suggesting greater vulnerability to ischemia-reperfusion injury. ${ }^{18}$

In this study, cyanotic patients had greater ventricular wall thickness, and inhomogeneous distribution of cardioplegic solution may be an important issue to provide adequate myocardial protection in hypertrophic hearts. ${ }^{1,19}$ Other clinical studies in pediatric patients undergoing cardiac surgery have demonstrated greater susceptibility to ischemia-reperfusion injury and worse clinical outcome in cyanotic patients. ${ }^{3}$ The hypoxic myocardium is also more susceptible to reoxygenation injury before and during cardiopulmonary bypass owing to free oxygen radicals. ${ }^{1,2}$ Increased pro-apoptotic genes and reduced expression of genes associated with myocardial contractility have been recently identified in cyanotic TOF. ${ }^{20}$

\section{HSP-70i Expression in Cyanotic Versus Acyanotic Myocardium}

HSP-70i limits ischemia-reperfusion injury by preserving mitochondrial function and inhibition of pro-apoptotic pathways through caspase-dependent and -independent pathways, thus promoting cardiomyocyte survival. ${ }^{6,21}$ Experimental induction of myocardial expression of HSP-70i by various laboratories means including heat-preconditioned, transgenic animals, and transfection models showed increased ischemic tolerance, reduced infarct size, and enhanced recovery of high-energy phosphate stores after ischemic insults. ${ }^{5,6,22}$

Acute ischemic insults were shown to trigger expression of HSP-70, but the effect of chronic hypoxemia in cyanotic subjects remained unclear. ${ }^{23}$ In the current study, in which the muscle was resected during TOF repair, we assumed the baseline HSP-70i concentration to represent the preoperative, steady state level in the patients. The inducible form of HSP-70i is expressed exclusively in response to adverse external stimuli; its presence in both noncyanotic and cyanotic myocardium might imply that chronic hypoxia was not a stimulus per se to the induction of HSP. There were 2 caveats: (1) we did not have a control group (ie, normal heart) in which the RV myocardium is exposed to normal pressures and with a fully oxygenated blood supply. It would be important to identify if HSP expression is different between in TOF and control. (2) The pressure-loaded $\mathrm{RV}$ in TOF may be a stimulus or the predominant stimulus to HSP induction, as has been demonstrated in animal studies. ${ }^{24}$ Similar to our findings that showed that total cellular HSP-70i was similar between cyanotic and noncyanotic subjects, Rafiee and associates ${ }^{25}$ identified an increased messenger RNA expression in cyanotic subjects and intracellular redistribution of HSP-70i from particulate to cytosolic compartment as part of the stress response to hypoxia. However, it was unclear whether these responses were due to cyanosis or the acute effect to any stress during surgery without an adequate time to allow protein manifestation.

\section{HSP-70i Response During Ischemic Cardioplegic Arrest}

In this study, RVOT muscle was resected at various ischemic intervals as part of the operative procedure to investigate the effect of increasing ischemic time on HSP expression. Within the limit of aortic crossclamp time, no effect of increasing ischemic duration and HSP expression was observed. This is in accordance with other studies examining HSP expression during cardioplegic arrest, and it is likely that there is not sufficient time for the protein upregulation during the RVOT resection period. ${ }^{8,26}$ Importantly, Vittorini and associates ${ }^{27}$ identified an increased HSP-70 gene expression within the duration of clamp time in children undergoing surgery. Although messenger RNA for HSP-70i is rapidly synthesized after external stimuli, the actual protein itself did not rise until much later. ${ }^{28}$ Other studies have confirmed that a protein elevation is seen only with a longer ischemic time of more than 2 hours. ${ }^{29}$ One can speculate that if it were possible to obtain biopsy specimens of the myocardium 24 hours after the ischemia-reperfusion stress, an increased expression of HSP might have been observed. 


\section{HSP-70i Induction and Clinical Implication}

In this study, a correlation between HSP-70i expression and improved postoperative RV function was observed, primarily in the cyanotic patients. Improved $\mathrm{S}_{\mathrm{v}} \mathrm{O}_{2}$ and its derived oxygen extraction factor also correlated with increased HSP$70 \mathrm{i}$ expression. These findings support previous animal studies that showed correlation between HSP and myocardial protection. ${ }^{5,6}$ Studies in patients undergoing open heart operations have similarly demonstrated increased HSP-70i expression associated with reduced biochemical markers of myocardial injury postoperatively in both adults and children. ${ }^{7,8}$ Taken together, these studies suggest that intracellular HSP-70i mediates protective effects against ischemia-reperfusion insult in the human myocardium. Of note in our study, the cardioprotective effect of HSP-70i was not seen in the noncyanotic group. The difference between the cyanotic and noncyanotic groups is conjectural but could be in part due to greater role of the HSP in cyanotic myocardium more susceptible to ischemia-reperfusion injury. There was also no correlation between HSP expression and troponin; however, this may be confounded by resection and direct surgical trauma, as previously discussed.

Various strategies have been studied to reduce the magnitude of ischemia-reperfusion injury, including ischemic preconditioning and HSP induction. ${ }^{5}$ The role of HSP induction to convey additional myocardial protection remains experimental and primarily investigated in animal models. Heat stress can be used in animal models but is not applicable in the clinical setting. Alternative stimuli such as transient ischemia, pharmacotherapy, or genetic manipulation may play a potential role. ${ }^{5}$ In addition to past studies that showed reduced myocardial injury with HSP-70i, this study identified the functional implication on early postoperative RV function and cardiac output in cyanotic TOF. Therapeutic induction of HSP-70i may be an option, possibly by preconditioning, or pharmacology might be a future option.

\section{LIMITATIONS}

As tissue samples were derived solely from the RVOT during TOF repair, it is not known whether the tissue's HSP-70 expression will be representative of the entire RV for 2 reasons: First, the infundibulum in the RV in TOF is the pathologic site subjected to chronic hypertrophic changes; second, the RVOT area is subjected to surgical trauma during muscle resection. The effect of type and timing of cardioplegia on HSP is also unknown, and its influence on HSP expression cannot be ruled out. The biopsy period was restricted to the crossclamp period, and there was probably insufficient time for protein expression to be realized.

\section{CONCLUSIONS}

RV dysfunction continues to be a short-term and longterm issue after repair of TOF, despite improvement in sur- gical technique and myocardial protection. In this congenital lesion, in which myocardium is exposed to chronic pressure loading and hypoxia, there may be an increased susceptibility to ischemia-reperfusion injury at the time of repair. HSP-70i, a chaperone protein expressed in response to cellular noxious stimuli, offers protection against injury, including ischemia-reperfusion. This study identified HSP-70i expression in the myocardium resected during repair of TOF in children. The presence of cyanosis was not associated with an increased expression of HSP-70i compared with that in acyanotic patients. No apparent increase in HSP-70i with increasing ischemic time was observed. In the cyanotic group, a significant correlation between HSP$70 \mathrm{i}$ expression and improved postoperative RV function as quantified by tissue Doppler annular velocities was evident. Surrogate markers of cardiac output, $\mathrm{S}_{\mathrm{v}} \mathrm{O}_{2}$, and derived oxygen extraction ratio were also improved with increased HSP-70i expression. From these findings we conclude that increased HSP-70i expression provides an important cardioprotective role in patients with cyanotic TOF undergoing surgical repair.

\section{References}

1. del Nido PJ, Mickle DA, Wilson GJ, Benson LN, Coles JG, Trusler GA, et al. Evidence of myocardial free radical injury during elective repair of tetralogy of Fallot. Circulation. 1987;76(5 Pt 2):V174-9.

2. Allen BS, Rahman S, Ilbawi MN, Kronon M, Bolling KS, Halldorsson AO, et al. Detrimental effects of cardiopulmonary bypass in cyanotic infants: preventing the reoxygenation injury. Ann Thorac Surg. 1997;64:1381-7; discussion 7-8.

3. Imura H, Caputo M, Parry A, Pawade A, Angelini GD, Suleiman MS. Agedependent and hypoxia-related differences in myocardial protection during pediatric open heart surgery. Circulation. 2001;103:1551-6.

4. Najm HK, Wallen WJ, Belanger MP, Williams WG, Coles JG, Van Arsdell GS, et al. Does the degree of cyanosis affect myocardial adenosine triphosphate levels and function in children undergoing surgical procedures for congenital heart disease? J Thorac Cardiovasc Surg. 2000;119:515-24.

5. Latchman DS. Heat shock proteins and cardiac protection. Cardiovasc Res. 2001;51:637-46.

6. de Jong PR, Schadenberg AW, Jansen NJ, Prakken BJ. Hsp70 and cardiac surgery: molecular chaperone and inflammatory regulator with compartmentalized effects. Cell Stress Chaperones. 2009;14:117-31.

7. Demidov ON, Tyrenko VV, Svistov AS, Komarova YY, Karpishenko AI, Margulis BA, et al. Heat shock proteins in cardiosurgery patients. Eur J Cardiothorac Surg. 1999;16:444-9.

8. Giannessi D, Caselli C, Vitale RL, Crucean A, Murzi B, Del Ry S, et al. A possible cardioprotective effect of heat shock proteins during cardiac surgery in pediatric patients. Pharmacol Res. 2003;48:519-29.

9. Chi NC, Karliner JS. Molecular determinants of responses to myocardial ischemia/reperfusion injury: focus on hypoxia-inducible and heat shock factors. Cardiovasc Res. 2004;61:437-47.

10. Mascio CE, Olison AK, Ralphe JC, Tomanek RJ, Scholz TD, Segar JL. Myocardial vascular and metabolic adaptations in chronically anemic fetal sheep. Am J Physiol Regul Integr Comp Physiol. 2005;289:R1736-45.

11. Piel DA, Khan AR, Waibel R, Birbach M, Cohen MS, Spray TL, et al. Chronic hypoxemia increases myocardial cytochrome oxidase. $J$ Thorac Cardiovasc Surg. 2005;130:1101-6.

12. Cullen S, Shore D, Redington A. Characterization of right ventricular diastolic performance after complete repair of tetralogy of Fallot. Restrictive physiology predicts slow postoperative recovery. Circulation. 1995;91:1782-9.

13. Lyall F, Barber A, Myatt L, Bulmer JN, Robson SC. Hemeoxygenase expression in human placenta and placental bed implies a role in regulation of trophoblast invasion and placental function. FASEB J. 2000;14: 208-19. 
14. Bradford MM. A rapid and sensitive method for the quantitation of microgram quantities of protein utilizing the principle of protein-dye binding. Anal Biochem. 1976;72:248-54.

15. Martin J, Shekerdemian LS. The monitoring of venous saturations of oxygen in children with congenitally malformed hearts. Cardiol Young. 2009;19:34-9.

16. Wernovsky G, Wypij D Jr, Mayer JE, Hanley F, Hickey P, Walsh AZ, et al. Postoperative course and hemodynamic profile after the arterial switch operation in neonates and infants. A comparison of low-flow cardiopulmonary bypass and circulatory arrest. Circulation. 1995;92:2226-35.

17. Plunkett MD, Hendry PJ, Anstadt MP, Camporesi EM, Amato MT, St Louis JD, et al. Chronic hypoxia induces adaptive metabolic changes in neonatal myocardium. J Thorac Cardiovasc Surg. 1996;112:8-13.

18. Silverman NA, Kohler J, Levitsky S, Pavel DG, Fang RB, Feinberg H. Chronic hypoxemia depresses global ventricular function and predisposes to the depletion of high-energy phosphates during cardioplegic arrest: implications for surgical repair of cyanotic congenital heart defects. Ann Thorac Surg. 1984;37:304-8.

19. del Nido PJ, Mickle DA, Wilson GJ, Benson LN, Weisel RD, Coles JG, et al. Inadequate myocardial protection with cold cardioplegic arrest during repair of tetralogy of Fallot. J Thorac Cardiovasc Surg. 1988;95:223-9.

20. Ghorbel MT, Cherif M, Jenkins E, Mokhtari A, Kenny D, Angelini GD, et al. Transcriptomic analysis of patients with tetralogy of Fallot reveals the effect of chronic hypoxia on myocardial gene expression. J Thorac Cardiovasc Surg. 2010;140:337-45. e26.

21. Suzuki K, Murtuza B, Sammut IA, Latif N, Jayakumar J, Smolenski RT, et al. Heat shock protein 72 enhances manganese superoxide dismutase activity during myocardial ischemia-reperfusion injury, associated with mitochondrial protection and apoptosis reduction. Circulation. 2002;106(12 Suppl. 1): I270-6

22. Okubo S, Wildner O, Shah MR, Chelliah JC, Hess ML, Kukreja RC. Gene transfer of heat-shock protein 70 reduces infarct size in vivo after ischemia/reperfusion in the rabbit heart. Circulation. 2001;103:877-81.

23. Dillmann WH, Mehta HB, Barrieux A, Guth BD, Neeley WE, Ross J Jr. Ischemia of the dog heart induces the appearance of a cardiac mRNA coding for a protein with migration characteristics similar to heat-shock/stress protein 71. Circ Res. 1986;59:110-4.

24. Osaki J, Haneda T, Kashiwagi Y, Oi S, Fukuzawa J, Sakai H, et al. Pressureinduced expression of heat shock protein $70 \mathrm{mRNA}$ in adult rat heart is coupled both to protein kinase A-dependent and protein kinase C-dependent systems. J Hypertens. 1998;16:1193-200.

25. Rafiee P, Shi Y, Pritchard KA Jr, Ogawa H, Eis AL, Komorowski RA, et al. Cellular redistribution of inducible Hsp70 protein in the human and rabbit heart in response to the stress of chronic hypoxia: role of protein kinases. $J$ Biol Chem 2003;278:43636-44.

26. McGrath LB, Locke M, Cane M, Chen C, Ianuzzo CD. Heat shock protein (HSP 72) expression in patients undergoing cardiac operations. J Thorac Cardiovasc Surg. 1995;109:370-6.

27. Vittorini S, Storti S, Andreani G, Giusti L, Murzi B, Furfori P, et al. Heat shock protein 70-1 gene expression in pediatric heart surgery using blood cardioplegia. Clin Chem Lab Med. 2007;45:244-8.

28. Mehta HB, Popovich BK, Dillmann WH. Ischemia induces changes in the leve of mRNAs coding for stress protein 71 and creatine kinase M. Circ Res. 1988;63: 512-7.

29. Schmitt JP, Schunkert H, Birnbaum DE, Aebert H. Kinetics of heat shock protein 70 synthesis in the human heart after cold cardioplegic arrest. Eur J Cardiothorac Surg. 2002;22:415-20.

\section{Discussion}

Dr James Jaggers (Denver, Colo). I appreciate the opportunity to comment. This is a very complex problem and you will agree that doing those experiments in clinical patients must be very difficult in uncontrolled and highly variable patients.

My only real question is this: What do you think the role the hypertrophy plays in the expression of HSP? There are multiple differences between your 2 groups, which I will not get into. Clearly, the cyanotic group was very different group from the noncyanotic group; but clearly the cyanotic group was significantly more hypertrophied. Can you speculate on the role of hypertrophy in this study on HSP?

Dr Danton. Heat shock proteins are upregulated in a variety of stress responses, including those that induce cardiac hypertophy. In this study, we did not specifically analyze the relationship between HSP expression and the magnitude of myocardial hypertophy. However, as you state, in patients with tetralogy of Fallot there are multiple factors which could induce HSP-hypoxia, RV hypertension, which may have a complex interaction and confound identification of HSP versus a single variable. 